\title{
Immediate Breast Reconstruction Using Silicone Implants in Previously Augmented Patients
}

\author{
Dong Hun Choi ${ }^{1}$, Dong Kyu Kim ${ }^{1}$, \\ Dong Wan Ryu ${ }^{1}$, Jeong Woo Lee ${ }^{1}$, \\ Kang Young Choi ${ }^{1}$, Ho Yun Chung ${ }^{1}$, \\ Byung Chae Cho ${ }^{1}$, Ho Yong Park ${ }^{2}$, \\ Jung Dug Yang ${ }^{1}$ \\ ${ }^{1}$ Department of Plastic and \\ Reconstructive Surgery, Kyungpook \\ National University School of Medicine, \\ Daegu; ${ }^{2}$ Departments of Surgery, \\ Kyungpook National University School of \\ Medicine, Daegu, Korea
}

No potential conflict of interest relevant to this article was reported.
Background Augmentation mammoplasty is rapidly becoming one of the most frequently performed cosmetic surgeries. Consequently, the number of breast cancer patients with a history of breast augmentation surgery will increase. The purpose of this study is to report our experience of breast reconstruction in augmented women and discuss their treatment characteristics.

Methods From March 2010 to August 2015, 7 patients who had previously undergone breast augmentation were treated at our institution. Epidemiologic data as well as data regarding body mass index, types of mastectomy, and complication were recorded and analyzed.

Results The mean age was 43.4 years, the average follow-up period was 21 months, and the mean body mass index (BMI) was $19.3 \mathrm{~kg} / \mathrm{m}^{2}$, the average weight of the mastectomy specimen was $150 \mathrm{~g}$. Implant-based immediate breast reconstruction after skin-sparing mastectomy was performed in seven patients and in four of these patients, acellular dermal matrix (ADM) was used. One patient had a capsular contracture of Baker grade II. One patient had seroma.

Conclusions Previously augmented patients tend to have a slender abdomen and back. Moreover, Asians have a smaller body frame and lower muscle and fat percentage than westerners, making it more suitable for implant-based reconstruction than autologous tissue. It can be recommended as a surgical method as our patients were highly satisfied with the cosmetic outcomes.

Keywords Breast neoplasms, Mammaplasty, Silicone gels

\section{INTRODUCTION}

Augmentation mammoplasty is one of the most frequently performed cosmetic surgeries worldwide. According to Cahan et al. [1], no statistical significance was noted in the mean tumor size, stage, nodal status of breast cancer between augmented patient group and non-augmented patients. Since the rate of breast cancer

Received: Jan 25, 2016 Revised: Feb 19, 2016 Accepted: Feb 20, 2016 Correspondence: Jung Dug Yang Department of Plastic and Reconstructive Surgery, Kyungpook National University School of Medicine, 130 Dongdeokro, Jung-gu, Daegu 41944, Korea. E-mail: lambyang@daum.net

Copyright @ 2016 The Korean Society for Aesthetic Plastic Surgery.

This is an Open Access article distributed under the terms of the Creative Commons Attribution Non-Commercial License (http://creativecommons.org/licenses/by-nc/3.0/) which permits unrestricted non-commercial use, distribution, and reproduction in any medium, provided the original work is properly cited. $\quad$ www.e-aaps.org is radically increasing, and breast augmentation surgery is performed actively over the recent years, the need for combined performance of breast cancer surgery and reconstruction in previously augmented patients is thus increasing $[2,3]$.

The anatomical and physical properties of the breast in patients with a history of mammoplasty differ from those in patients who have not undergone mammoplasty. Moreover, the patient's high interest in aesthetic appearance and having significantly less breast tissue shows the difference from those patients who have not undergone mammoplasty.

Patients who have undergone breast augmentation commonly have a low body mass index (BMI), lesser lower abdominal and back tissue limits in harvesting autologous tissues [4]. Meanwhile, skin-sparing mastectomy enables immediate implant-based breast reconstruction. By utilizing the existing implants inserted space, new implants can be inserted more easily. We report our experi- 
ence of treating breast cancer patients after breast augmentation with skin-sparing mastectomy and breast reconstruction with satisfactory results.

\section{METHODS}

An individual plastic surgeon performed breast augmentation to total of seven patients from March 2010 to August 2015. Patient's Information regarding underlying disease, age, BMI, smoking history, irradiation history, weight and skin surface of the excised breast during surgery, postoperative complications, reoperation, usage of acellular dermal matrix (ADM), and sizes and kinds of replaced implants were obtained from the medical records. Information regarding postoperative chemotherapy and radiotherapy were additionally obtained. Six months after the surgery, we followed up with the level of cosmetic satisfaction including breast symmetry and overall cosmetic effectiveness with both patients and the surgeon.

As a surgical method, first skin-sparing or nipple sparing mastectomy was performed by an oncologic breast surgeon, and a plastic surgeon removed the already inserted implant in subpectoral or subglandular plane. Capsulectomy was performed if it was placed in subglandular plane and a new implant was inserted after a space

Table 1. KNUH breast reconstruction satisfaction questionnaire

\begin{tabular}{|c|c|c|c|c|c|}
\hline Question & Very dissa & isfied & $\rightarrow$ & Very satisfied & Mean score \\
\hline Q1. Overall satisfaction with my breast reconstruction & 1 & 2 & 3 & 4 & 4.31 \\
\hline Q2. Symmetry of my breasts & 1 & 2 & 3 & 4 & 4.24 \\
\hline Q3. Size of my reconstructed breast & 1 & 2 & 3 & 5 & 4.41 \\
\hline Q4. Shape of my reconstructed breast & 1 & 2 & 3 & 5 & 4.37 \\
\hline Q5. Feel to touch my reconstructed breast & 1 & 2 & 3 & 4 & 3.93 \\
\hline Q6. Pain in my reconstructed breast & 1 & 2 & 3 & 5 & 4.18 \\
\hline Q9. Donor site scar & \multicolumn{4}{|c|}{$\mathrm{N} / \mathrm{A}$} & - \\
\hline Q10. Self-confidence & 1 & 2 & 3 & 4 & 4.28 \\
\hline Q11. Sexual attractiveness & 1 & 2 & 3 & 4 & 4.31 \\
\hline
\end{tabular}

N/A, not available.
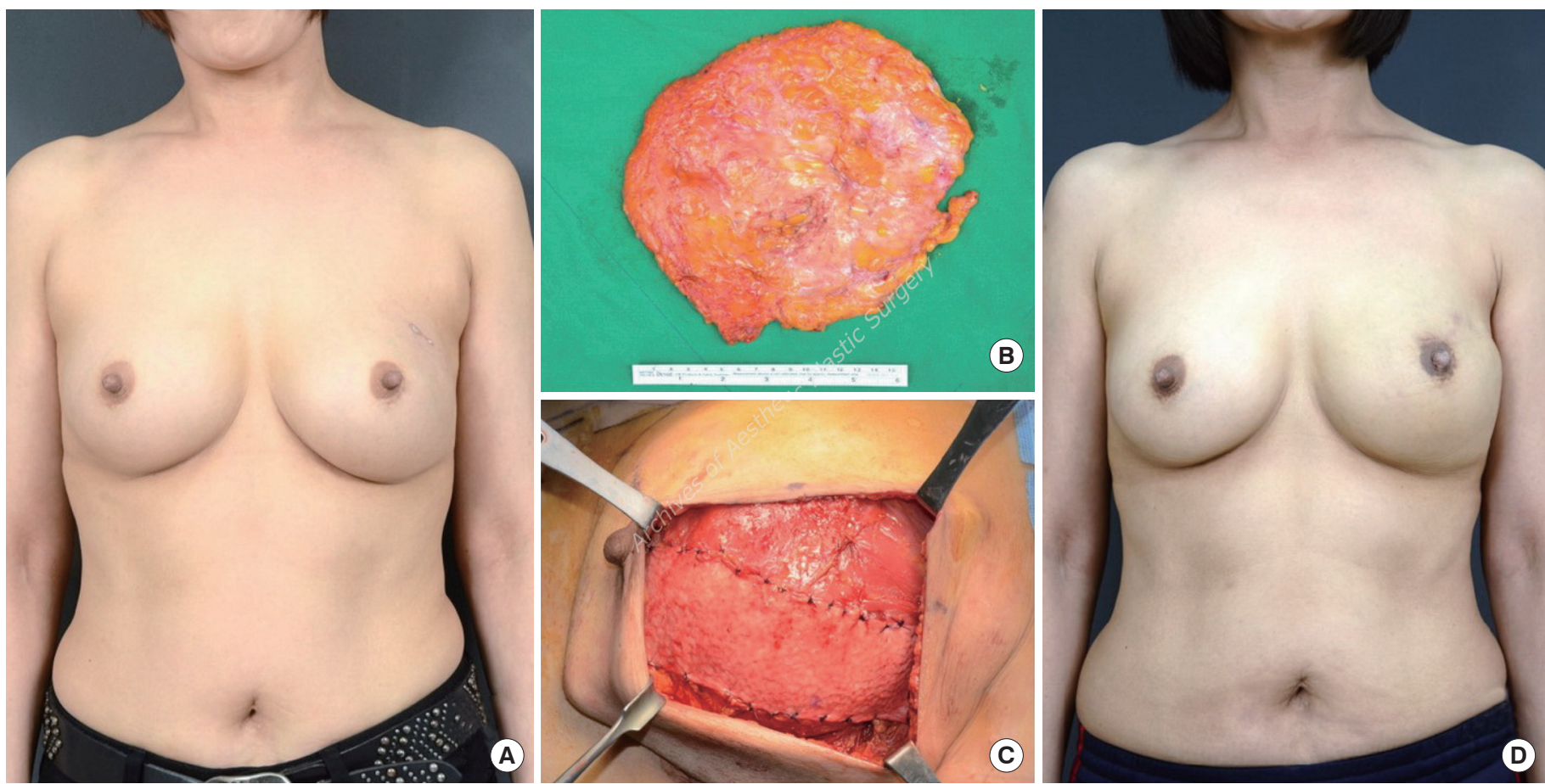

Fig. 1. A 54-year-old woman with lobular carcinoma in situ (LCIS) in left upper outer breast. (A) Preoperative view. (B) Tissue after skin sparing mastectomy. (C) Intraoperative view of changed implant with acellular dermal matrix (ADM; sling) in the pocket after skin sparing mastectomy. (D) 10 months postoperative outcome. 

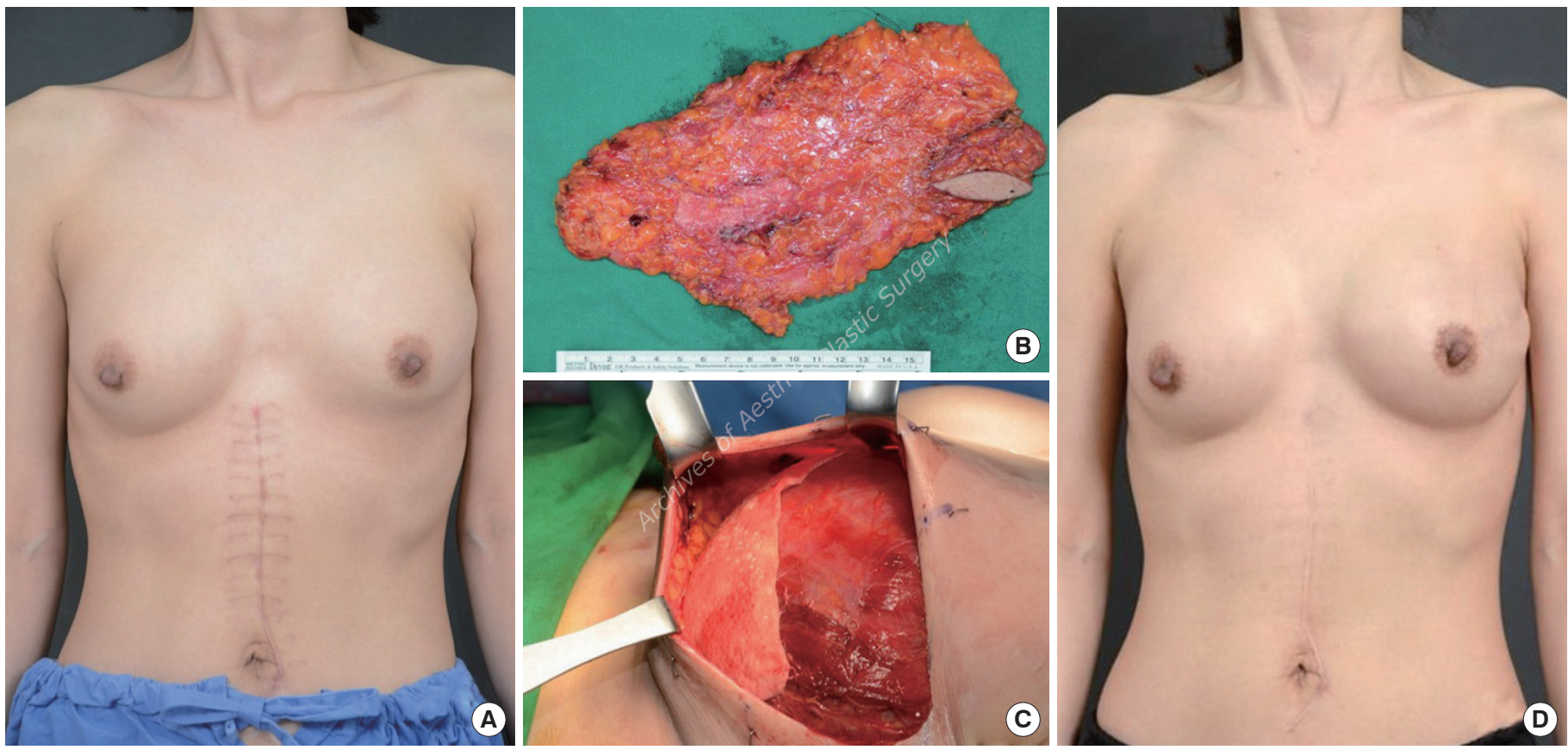

Fig. 2. A 39-year-old woman with ductal carcinoma in situ (DCIS) in left upper outer breast. (A) Preoperative view. (B) Tissue after skin sparing mastectomy. (C) In ora-operative view of changed implant with acellular dermal matrix (ADM; onlay graft) in the pocket after skin sparing mastectomy. (D) 18 months postoperative outcome.

to insert an implant was created by dissecting subpectoral area. ADM covered the implant using a sling for pectoralis muscle. When an implant was located in the subpectoral plane, the area of previous adhesion was dissected after the implant was removed. Then, capsulectomy was performed and thus an enough space for the insertion of a new implant was created. In one case, onlay graft of ADM was performed in order to prevent irregularity and dimpling, which develops as breast skin flap is thin. In order to prevent post-operative infection, sufficient irrigation was performed using the mixed solution of antibiotics and potadine. For a new implant, the approximate size was predicted by measuring the pre-operative breast volume and width of a patient. Then, the implant size was selected when it was most symmetric with the opposite side in a sitting position once a sizer was applied during surgery.

Referencing Kyungpook National University Hospital (KNUH) breast reconstruction satisfaction questionnaire (A total of 11 items), a survey was conducted among patients 6 months after the surgery regarding overall and cosmetic satisfaction (Table 1). Each item was graded on a 5-point scale from highly dissatisfied (1) to highly satisfied (5). Three plastic surgeons evaluated the postoperative photographs (6 months) from very bad (1) to very good (5) in accordance with Likert scale.

\section{RESULTS}

A total of seven patients with a mean age of 43.4 years (range, 31-
54 years) were investigated from March 2010 to August 2015. The mean follow up period by the outpatient clinic was 21 months (range, 6-31 months). The mean BMI was $19.3 \mathrm{~kg} / \mathrm{m}^{2}$ (range, 17.5-21.9) and the mean excised mass weight was $150 \mathrm{~g}$ (range, 68-260 g). Four of the patients existing breast implant location was at the subpectoral plane, and the rest three patients were at the subglandular plane. Implants were replaced after mastectomy. A total of four patients had implants covered by ADM; where three of these patients had implants placed in subpectoral and subgraft pocket with ADM acting as a sling (Fig. 1). One patient underwent onlay graft to prevent depression and irregularity due to a thin breast skin flap after skin-sparing mastectomy (Fig. 2). In terms of tumor diagnosis, one patient had lobular carcinoma in situ, three had invasive ductal carcinoma, and three had ductal carcinoma in situ. Baker grade II capsular contracture was developed in 1 case, and 1 case of seroma was noted after surgery. Characteristics of patients are shown in Table 2. The mean patient satisfaction score was 4.27 point, and the mean satisfaction score rated by plastic surgeons was 4.21 points.

\section{DISCUSSION}

Augmentation mammoplasty is currently one of the most commonly performed cosmetic surgeries [5]. There has been a 5-fold increase in the number of breast augmentation surgeries in the US, similarly, there has been a consistent increase in the number of breast augmentation surgeries in South Korea [2,3]. Irrespectively, 
the number of breast cancer cases is increasing; in South Korea, there were 16,967 patients with breast cancer in 2011, which was a 4 -fold increase compared to the number of patients in 1996. This indicates that the possibility of developing breast cancer is increasing in patients who have undergone breast augmentation [6].

In patients who had undergone breast augmentation, the tumor was smaller, was more palpable, and had a lower clinical stage than that in patients who had not undergone breast augmentation. Moreover, previously augmented patients were younger at the time of diagnosis. It is known that implants do not influence estrogen receptor expression and lymph node metastasis. Furthermore, the survival rate and the local recurrence rate did not differ significantly between patients who have and have not undergone augmentation [7].

Generally, two surgical treatments are available for removing the tumor in previously augmented patients: breast-conserving surgery and mastectomy [4]. According to Singh et al. [8], patients who have undergone augmentation mammoplasty are not ideal candidates for breast-conserving surgery owing to its small breast size, and may experience distortion after lumpectomy. Handel et al. [9] reported that augmented breast cancer patients treated with breast conserving surgery have less cosmetic satisfaction. Moreover, mammographic follow-up for detection of the tumor recurrence may be impaired by the implants. The possibility of capsular formation may also increase after postoperative radiotherapy. Therefore, reconstruction by implants has cosmetic and oncologic limitations when breast conserving surgery is performed.

When performing mastectomy in patients with previously augmented breasts, the implant itself does not hinder the surgery as implants, scar, or capsules can be excised. If skin-sparing mastectomy is performed, it is possible to conduct one-stage breast reconstruction by replacing the existing one with new implants after mastectomy. If skin-sparing breast reconstruction is performed, it is possible to maintain the inframammary fold, normal skin color, texture, and sensation, and to decrease the size of scar [10]. Therefore, breast reconstruction using implants in patients with skin-sparing mastectomy is considered a better option that breast-conserving surgery.

An augmented breast normally requires more volume to maintain symmetry, as the controlateral breast is more enlarged than one's own breast. However, patients who have undergone augmentation mammoplasty usually have low BMI and have slender abdomen and back. Especially for Asians tend to have smaller body frame with lower muscle and fat percentage compared to westerners, the usage of autologous tissue is restricted. The average BMI of the 7 patients we operated was $19.3 \mathrm{~kg} / \mathrm{m}^{2}$, which limited the use of autologous tissue for defects that had occurred after skin-sparing mastectomy. Therefore, with the excised amount of breast tissue considered, we replaced it with a bigger implant to minimize volume difference from a controlateral breast [11]. 
According to Robbins et al. [12], indications for implant removal during mastectomy are as follows: implants in the subglandular position, rupture, infection, extrusion or expose of implants, capsular contracture, pain, seroma, outdated implants, cosmetic dissatisfaction, implants located in proximity to a tumor, and cases requiring irradiation. In all 7 patients of this study, a new implant was placed after previous implant was removed; In 3 patients, the implants were replaced, its location was altered from the subglandular to the subpectoral position, and the implants were covered by slinging ADM. In the rest of 4 patients, previous implants were removed due to cosmetic dissatisfaction and then new implants were inserted to make symmetry.

Roostaeian et al. [13] reported cases of complications that numbered 18 of 38 (63 breasts) with a history of augmentation and 20 of 77 (138 breasts) patients who had not undergone breast augmentation. Capsular contracture occurred most commonly, followed by wound dehiscence, hematoma, and infection. In our case, two among seven patients developed post-operative complications; One patient had Baker grade II capsular contracture and another had seroma.

Using the Breast Q, Salgarello et al. [11] investigated the level of satisfaction with reconstruction using implants in patients who had and had not undergone breast augmentation and found that those who had undergone breast augmentation previously showed higher satisfaction levels. Spear et al. [14] reported similar results. In this study, the patient group was small and thus it was not statistically significant. Also, there is a limitation that there is no appropriate control group, but the mean score of satisfaction was measured as relatively high. Using a breast reconstruction satisfaction questionnaire in our study, we reported a mean satisfaction score of 4.27 among patients and 4.21 among plastic surgeons, which are relatively high values. Patients who have undergone augmentation mammoplasty are concerned about cosmetic outcomes such as the breast size and shape, and they remained very satisfied after surgery because the breast shape was maintained.

This study, despite the small number of patients enrolled, achieved satisfying results in breast reconstruction using implants for breast cancer patients who had undergone breast augmentation previously, our findings are especially applicable in Asians and further studies are required to determine other populations where our findings may be applicable.

\section{CONCLUSIONS}

Since augmentation mammoplasty is performed actively worldwide, the need for surgery of breast cancer and reconstruction is also increasing from the patients with a history of breast augmentation. We reported seven cases showing that immediate implantbased reconstruction is suitable for previously augmented patients when skin-sparing mastectomy is performed. It is a good surgical option as our patients were highly satisfied with the cosmetic results.

\section{PATIENT CONSENT}

Patients provided written consent for the use of their images.

\section{REFERENCES}

1. Cahan AC, Ashikari R, Pressman P, et al. Breast cancer after breast augmentation with silicone implants. Ann Surg Oncol 1995;2:121-5.

2. Siegel R, Ma J, Zou Z, et al. Cancer statistics, 2014. CA Cancer J Clin 2014;64:9-29.

3. Korean Breast Cancer Society. Breast cancer facts \& figures 2014. Seoul: Korean Breast Cancer Society; 2014.

4. McCarthy CM, Pusic AL, Disa JJ, et al. Breast cancer in the previously augmented breast. Plast Reconstr Surg 2007;119:49-58.

5. American Society of Plastic Surgeons. 2014 cosmetic plastic surgery statistics; 2015 [cited 2016 Feb 14]. Available from http://www.plasticsurgery.org/Documents/news-resources/statistics/2014-statistics/cosmetic-procedure-trends-2014.pdf.

6. Tuli R, Flynn RA, Brill KL, et al. Diagnosis, treatment, and management of breast cancer in previously augmented women. Breast J 2006; 12:343-8.

7. Hölmich LR, Mellemkjaer L, Gunnarsdóttir KA, et al. Stage of breast cancer at diagnosis among women with cosmetic breast implants. $\mathrm{Br} \mathrm{J}$ Cancer 2003;88:832-8.

8. Singh KA, Saunders N, Carlson GW. Immediate breast reconstruction in the previously augmented patient. Ann Plast Surg 2012;68:477-80.

9. Handel N, Lewinsky B, Jensen JA, et al. Breast conservation therapy after augmentation mammaplasty: is it appropriate? Plast Reconstr Surg 1996;98:1216-24.

10. Toth BA, Lappert P. Modified skin incisions for mastectomy: the need for plastic surgical input in preoperative planning. Plast Reconstr Surg 1991;87:1048-53.

11. Salgarello M, Rochira D, Barone-Adesi L, et al. Immediate breast reconstruction after skin- or nipple-sparing mastectomy for previously augmented patients: a personal technique. Aesthetic Plast Surg 2012; 36:313-22.

12. Robbins CM, Long JN, Fix RJ, et al. Mastectomy with breast reconstruction in previously augmented patients: indications for implant removal. Ann Plast Surg 2008;61:500-5.

13. Roostaeian J, Yoon AP, Rahgozar P, et al. Implant-based immediate breast reconstruction in the previously augmented patient. J Plast Reconstr Aesthet Surg 2015;68:e71-9.

14. Spear SL, Slack C, Howard MA. Postmastectomy reconstruction of the previously augmented breast: diagnosis, staging, methodology, and outcome. Plast Reconstr Surg 2001;107:1167-76. 